\title{
Economic security evaluation model of enterprises based on the latent variables theory
}

\author{
Sergey Barkalov ${ }^{1}$, Sergey Moiseev ${ }^{1, *}$, Vera Poryadina $^{1}$, and Denis Kvasov ${ }^{1}$ \\ ${ }^{1}$ Voronezh State Technical University, Moskovsky Prospect, 14, Voronezh, 394026, Russia
}

\begin{abstract}
The paper presents the model of obtaining a comprehensive assessment of economic security for a group of enterprises. A comprehensive assessment of enterprise ES is based on the assessment of measures for some components of ES. The work highlights the components: financial, information, personnel, technical and technological, legal and power. Much attention is given to the traditional estimation method and an alternative approach based on the Rasch model for estimating latent variables. A comparison of the estimates of ES obtained by different methods is carried out, and using computational experiments, the adequacy and objectivity of the estimates obtained by the method proposed in the work are established. In addition, it was shown that a model based on the theory of latent variables has the following main advantages over the additive model, which follow from the properties of the Rasch model: comprehensive assessments of enterprise ES are their individual characteristics and are independent of the set of components for which the assessment is carried out; EB scores are measured on a linear dimensionless scale that can be easily converted to any other scale; in addition to comprehensive assessments of the ES of enterprises it is possible to obtain estimates of the ES components obtained for the entire group of enterprises, which are also individual for the components and linear.
\end{abstract}

\section{Introduction}

Economic security (ES) of an enterprise refers to the level of protection of vital aspects of the economic activity of an enterprise from internal and external threats by implementing measures of legal, financial, informational, organizational, engineering and other areas.

The concept of economic security is quite young, but nowadays it is often used to assess the state of economic stability of enterprises and organizations. Economic security is an integrated assessment of many components of the efficiency of economic activities of enterprises from the point of view of various aspects of its functioning.

The problem of obtaining comprehensive quantitative assessments of the economic security of enterprises and organizations is one of the most relevant in this area of economic research. In this article, the task is to analyze the traditional methods of assessing the state of economic security for the whole set of its components, to identify their advantages and

*Correspondent: mail@moiseevs.ru 
disadvantages, and to propose a new approach to a comprehensive assessment of the level of economic security for a group of enterprises in the approach of the theory of latent variables. Here are some aspects of the methodology for assessing the economic security of enterprises and organizations.

\section{Components of economic security of enterprises}

Actually, the concept of "economic security" is a new term, although the issues of its provision, prevention of threats and disasters have a long history of socio-economic theoretical research. Actually, the term "economic security" began to be actively used in scientific and everyday life abroad only in the mid 30s of the 20th century, after the widely known appeal to the nation by US President Franklin Roosevelt, and this term was used in the context of providing national ES.

This approach is still ongoing $[1,2]$. In Russia the category of economic security at the legislative level was first designated in 1992. The RF Law “About Security" established the concepts of security, objects and subjects of security, threats to security and providing security.

A comprehensive assessment of enterprise ES is based on the assessment of measures for some components of ES. According to [2], There are six main components.

The financial component - is a set of measures to ensure the highest level of solvency of the enterprise and the liquidity of its working capital, an effective capital structure [3].

The information component includes the functions of the information and analytical department necessary to achieve an acceptable level of information security.

The personnel component is work with personnel related to the prevention of threats due to insufficient qualifications of employees, poor organization of the personnel management system, selection, training and motivation of employees [4].

The technical and technological component includes measures aimed at meeting the level of technology used at the enterprise with international standards

The legal component includes the effective legal support of the enterprise, compliance with it and its employees the legal norms of applicable law.

The power component is the physical safety of the employees of the enterprise, the safety of the property of the enterprise, preventive measures to the sources of possible environmental threats.

However, there are often approaches where they concretize the components of ES described above, breaking them into parts. But it is immediately worth mentioning that the models presented in the work are not tied to a certain number of components and are applicable to any number of them.

A comprehensive assessment of the ES of enterprises is built on the basis of private assessments of the components of the ES and they are obtained on the basis of objective indicators of the economic activity of enterprises or on the basis of expert assessment. In this article we describe the models for obtaining complex estimates of ES based on estimates of its components and do not pay attention to how particular estimates of the components were obtained and in what units and on what scales they are measured.

In view of this the model should be universal, independent of component estimates. We are considering two such models - the additive, traditionally used to solve such problems, and the original, based on the theory of latent variables. 


\section{An additive model of a comprehensive assessment of economic security}

The simplest model for obtaining a comprehensive assessment of ES for a group of enterprises is based on component estimate and is usually used to solve such problems. According to this model, a comprehensive assessment of the ES is obtained by summing up the partial estimates of the components, therefore, the model is called additive. Let's consider a mathematical model for solving the problem.

The attention is given to a group of $\mathrm{N}$ enterprises, which $\mathrm{ES}$ we need to estimate: $E_{1}, E_{2}$, $\ldots, E_{N}$. Evaluation is based on private estimates for $M$ component: $C_{1}, C_{2}, \ldots C_{M}$. We denote assessment of $i$ enterprise by $j$ component of ES by $U_{i j}$.

These estimates can be of different dimensions and measured on different scales. In order to compare them and obtain a comprehensive assessment of ES particular estimates of the components should be uniformed, therefore, at the first stage, it is necessary to normalize them to a single scale, for example, a unit one. To do this we propose the following normalization algorithm [5,6], as a result of which all normalized assessments of enterprises by the components of electronic components, which we denote, will take values from the interval $(0 ; 1)$ :

$$
u_{i j}=\frac{U_{i j}-\min _{i}\left(U_{i j}\right)}{\max _{i}\left(U_{i j}\right)-\min _{i}\left(U_{i j}\right)} .
$$

You can use other normalization algorithms that allow you to transfer estimates to a unit scale.

It should also be taken into account that when receiving a comprehensive assessment of ES, its components can give different contributions to the final assessment. To take this into account, we introduce the weights $w_{j}$, which make sense of the importance of the components of ES for this group of enterprises.

After that a comprehensive assessment of the enterprise ES $E_{i}$ can be obtained by the formula:

$$
X_{i}=\sum_{j=1}^{M} w_{j} u_{i j} ; \quad i=1,2, \ldots, N
$$

The additive method of finding a comprehensive assessment of ES (2) is the simplest, therefore, it is often used in practice. However, it has a number of significant drawbacks, in particular, the estimates do not take into account the features of the estimates of the components, depend on the set of enterprises being evaluated and are interdependent. In view of this, hereinafter we present a method for obtaining complex estimates of enterprise ES based on the Rasch model for estimating latent variables.

\section{A model for obtaining a comprehensive assessment of economic security based on the theory of latent variables}

Let's consider a group of $\mathrm{N}$ enterprises whose ES need to be estimated on the basis of private estimates for $\mathrm{M}$ components. There are assessments of enterprises normalized to a single scale by the components of the ES $u_{i j}$. 
To complete the task, we will use the theory of latent variables or rather the Rasch model for evaluating latent variables $[7,8]$. The Rasch model has a number of properties, which allows it to be used for a comprehensive assessment of enterprise ES with the correction of the deficiencies of the additive method described above. Let us list the advantages of the Rasch model:

1.The Rasch model allows you to convert measurements made in various scales (including qualitative) into linear measurements, as a result of this, quantitative methods can be used for qualitative data.

2. Due to the fact that the scale of measurement of estimates obtained on the basis of the Rasch model is linear, various transformations and statistical methods can be applied to the obtained estimates.

3. Assessment of the state of the enterprise's ES, obtained on the basis of the Rasch model, does not depend on the set of ES components and is an individual characteristic of each enterprise.

4. Along with comprehensive assessments of the status of ES, the model allows you to obtain assessments of the status of each component of ES for the entire group of enterprises, which allows for general monitoring of the level of ES for each component. Moreover, these estimates of the components are also independent of many enterprises and are independent.

We proceed directly to the estimation model. We introduce the following latent variables:

$\theta_{i}$ - a comprehensive indicator of the state of the enterprise's ES $E_{i}$, similar to the assessment of $X_{i}$ from formula (2);

$\beta_{j}$ - the integral assessment of the component of ES $C_{j}$ for the entire group of enterprises, the smaller this indicator, the greater the assessment of this component for all enterprises.

In accordance with the Rasch model, the likelihood that the enterprise $E_{i}$ has an estimate of component $C_{j}$ is higher than the estimate of this component for all enterprises is determined by the logistic function:

$$
P_{i j}=\frac{e^{\theta_{i}-\beta_{j}}}{1+e^{\theta_{i}-\beta_{j}}} .
$$

Assessments $\theta_{i}$ and $\beta_{j}$ are calculated based on known private estimates $u_{i j}$ by Model Rasch, based on the least squares method $[9,10]$ : latent variables $\theta_{i}$ and $\beta_{j}$ from model (3) are selected based on the condition of minimizing the sum of squared deviations of empirical estimates $u_{i j}$ from theoretical probabilities (3). Given the weights of the ES $w_{j}$, mathematically finding latent variables reduces to solving a nonlinear optimization problem of the form:

$$
\sum_{i=1}^{N} \sum_{j=1}^{M} w_{j}\left(u_{i j}-P_{i j}\right)^{2}=\sum_{i=1}^{N} \sum_{j=1}^{M} w_{j}\left(u_{i j}-\frac{e^{\theta_{i}-\beta_{j}}}{1+e^{\theta_{i}-\beta_{j}}}\right)^{2} \rightarrow \min .
$$

Estimates of the latent variables $\theta_{i}$ and $\beta_{j}$ obtained from the solution of problem (4) will be measured on interval and linear scales, but the reference point in them is not defined. The initial level of the reference scales, you can select the zero level of the rating scale so that the values $\theta_{i}$ and $\beta_{j}$ of all ratings were non-negative. Then the objective function (4) will be supplemented by the conditions: 


$$
\theta_{i} \geq 0 ; \beta_{j} \geq 0 ; i=1,2, \ldots, N ; \quad j=1,2, \ldots, M
$$

After receiving comprehensive estimates of ES, you can also normalize them, for example, they can be normalized to a scale at which the value of a comprehensive assessment of ES will be equal to a fraction in the unit sum of all estimates. Normalized estimates $\widetilde{\theta}_{i}$ are obtained from found $\theta_{i}$ by the formula:

$$
\widetilde{\theta}_{i}=\frac{\theta_{i}}{\sum_{i=1}^{N} \theta_{i}} .
$$

Based on these assessments, it is possible to rank enterprises according to the degree of their ES and also evaluate the components of electronic information for the entire group of enterprises.

\section{Properties of assessments of economic security of enterprises}

In order to verify the adequacy of the estimates of ES using a model based on the theory of latent variables, as well as to analyze the properties of estimates, computational experiments were carried out with input data matrices uij of various sizes, the elements of which were generated randomly, including taking into account the weights of the components of the ES.

The calculations were carried out in MS Excel using "Solver". The technique for solving such problems in Excel is described in [10]. An example of one of these calculations is given below.

Suppose that there are 10 enterprises whose ES are evaluated by 6 ES components. The weights (importance) of the components of ES for this example will be considered equal and we will take them unit. Let the matrix of normalized estimates of enterprises' ES by components be given in Table 1

Table 1. An example of normalized data for evaluating the EB of enterprises in its components.

\begin{tabular}{|c|c|c|c|c|c|c|c|c|c|c|}
\hline \multirow{2}{*}{$\begin{array}{c}\text { Component } \\
\text { ES }\end{array}$} & \multicolumn{10}{|c|}{ Enterprise } \\
\cline { 2 - 12 } & $\mathbf{E}_{\mathbf{1}}$ & $\mathbf{E}_{\mathbf{2}}$ & $\mathbf{E}_{\mathbf{3}}$ & $\mathbf{E}_{\mathbf{4}}$ & $\mathbf{E}_{\mathbf{5}}$ & $\mathbf{E}_{\mathbf{6}}$ & $\mathbf{E}_{\mathbf{7}}$ & $\mathbf{E}_{\mathbf{8}}$ & $\mathbf{E}_{\mathbf{9}}$ & $\mathbf{E}_{\mathbf{1 0}}$ \\
\hline $\mathrm{C}_{1}$ & 0.42 & 0.95 & 0.28 & 0.82 & 0.21 & 0.41 & 0.42 & 0.78 & 0.82 & 0.26 \\
\hline $\mathrm{C}_{2}$ & 0.23 & 0.58 & 0.51 & 0.14 & 0.50 & 0.08 & 0.52 & 0.41 & 0.51 & 0.23 \\
\hline $\mathrm{C}_{3}$ & 0.06 & 0.72 & 0.69 & 0.06 & 0.07 & 0.36 & 0.33 & 0.10 & 0.75 & 0.75 \\
\hline $\mathrm{C}_{4}$ & 0.18 & 0.31 & 0.87 & 0.55 & 0.70 & 0.23 & 0.36 & 0.60 & 0.12 & 0.26 \\
\hline $\mathrm{C}_{5}$ & 0.28 & 0.72 & 0.28 & 0.33 & 0.14 & 0.91 & 0.34 & 0.21 & 0.06 & 0.17 \\
\hline $\mathrm{C}_{6}$ & 0.57 & 0.38 & 0.82 & 0.13 & 0.42 & 0.75 & 0.43 & 0.90 & 0.87 & 0.92 \\
\hline
\end{tabular}

As a result of the calculation of complex estimates of enterprises' ES by the additive method and the method based on the Rasch model, the results are shown in Fig. 1. 


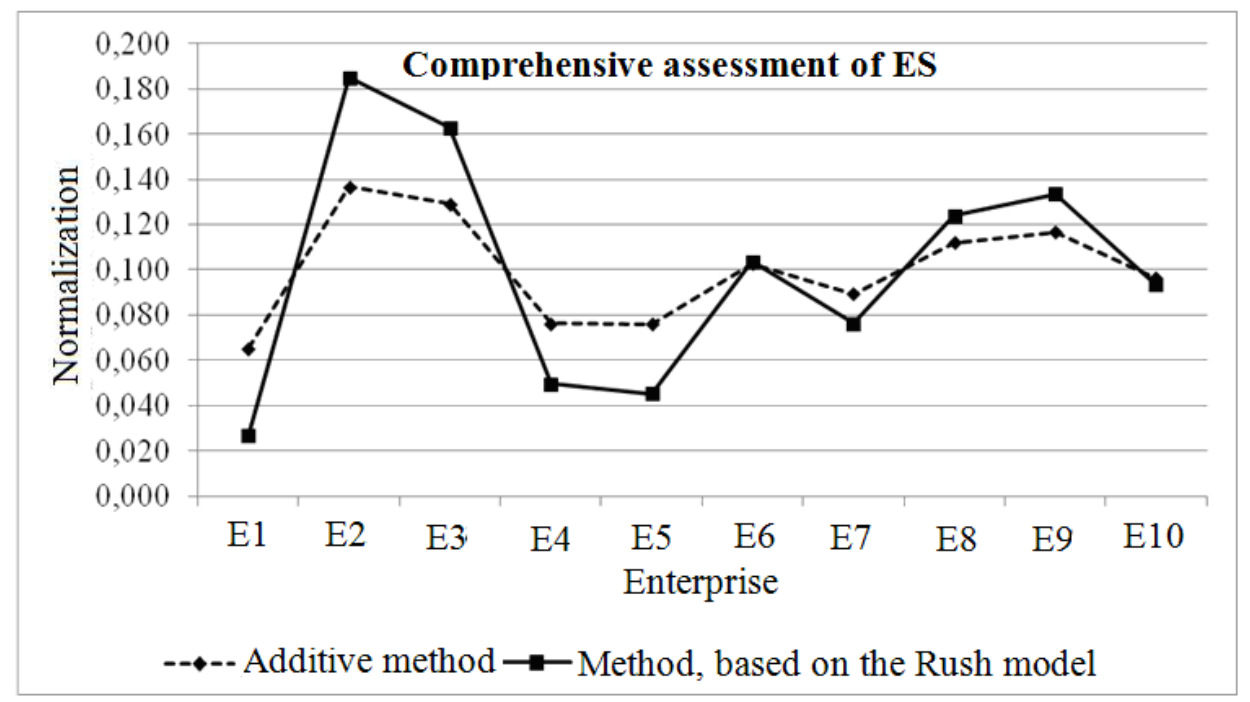

Fig. 1. Normalized by formula (6), comprehensive assessments of the enterprises state ES obtained by different methods according to the data in Table one.

Fig. 1 shows that the results of evaluations obtained using the Rasch model are in good agreement with the results of evaluations using the additive method. The average Pearson correlation coefficient for all computational experiments is 0.981 , for this example 0.998 .

The consistency of the estimates according to the Rasch model with the traditional and tested additive method indicates that the proposed method for evaluating the ES, based on the Rasch model for assessing latent variables, provides adequate comprehensive estimates of the ES of enterprises, which makes it possible to use it in practical research.

The expediency of using the Rasch model to solve problems of this type is due to the quality advantages of the estimates obtained, which are described earlier. In addition, the presented model makes it possible to obtain estimates of the components of ES for the entire group of enterprises being evaluated.

These estimates, for example, the data from the table. 1 are shown in Fig. 2, where the estimates are inverted and normalized by the formula (6). With this approach to rationing, the estimates of the components are the greater, the better the final estimates of each component for the entire set of enterprises being evaluated.

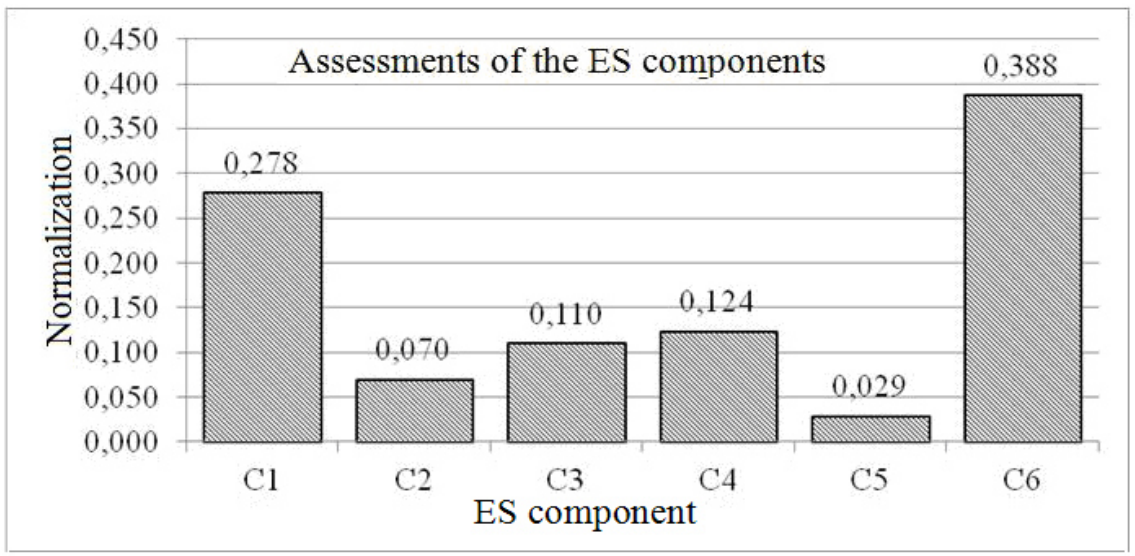

Fig. 2. Estimates of the ES components obtained by the Rasch model. 
Fig. 2 shows that for this group of enterprises the most secure is component $\mathrm{C} 5$, and the most problematic is $\mathrm{C} 3$. These estimates will allow us to optimally identify weaknesses in terms of ES for this group of enterprises and organizations and carry out preventive measures to improve ES.

\section{Conclusion}

Thus, the paper presents a model for obtaining a comprehensive assessment of ES for a group of enterprises, obtained on the basis of estimates of the components of ES. The model is based on the Rasch method for evaluating latent variables. Also described is the traditional additive model for assessing economic security, based on the weighted summation of particular estimates of the components of ES.

A comparison of the estimates of ES obtained by different methods is carried out, and using computational experiments, the adequacy and objectivity of the estimates obtained by the method proposed in the work are established.

In addition, it was shown that a model based on the theory of latent variables has the following main advantages over the additive model, which follow from the properties of the Rasch model [11]:

Comprehensive assessments of enterprise ES are their individual characteristics and are independent of the set of components for which the assessment is carried out.

EB scores are measured on a linear dimensionless scale that can be easily converted to any other scale.

In addition to comprehensive assessments of the ES of enterprises it is possible to obtain estimates of the ES components obtained for the entire group of enterprises, which are also individual for the components and linear. These estimates make it possible to additionally monitor the state of the ES from the point of view of dividing it into components for the entire set of enterprises, identifying those components for which it is first necessary to carry out preventive measures to increase the overall level of ES.

The described model will allow more efficient objective monitoring of the level of ES for a certain group of enterprises or organizations.

\section{References}

1. M.A. Soynikov, Contemporary Problems of Social Work 1.1 (1), 176-183 (2015)

2. Z. Varnaliy, S. Onyshchenko, O. Masliy, Science Rise 7.1(24), 41-46 (2016)

3. S.A. Barkalov, O.S. Perevalova, T.A. Averina, Development of the Algorithm for Maximizing the Financial Results of the Investment Program, 8551863 (2018)

4. V.V. Burkov, I.V. Burkova, T.A. Averina, T.V. Nasonova, Proceedings of 2017 10th International Conference Management of Large-Scale System Development, MLSD 2017, 8109602 (2017)

5. A.A. Maslak, S.I. Moiseev, T.A. Nasonova, Society, Integration, Education, Proceeding of the International Scientific Conference, Rezekne Academy of Technologies VI, 330-340 (2018)

6. A. Maslak, S. Pozdniakov, Society, Integration, Education, Proceeding of the International Scientific Conference, Rezekne Academy of Technologies I, 373-383 (2018)

7. G. Rasch, Probabilistic Models for Some Intelligence and Attainment Tests (Danish Institute for Educational Research, Denmark, 1960) 
8. S.A. Barkalov, S.I. Moiseev, O.N. Bekirova, Proceedings of 2018 11th International Conference "Management of Large-Scale System Development", MLSD 2018 (Moscow, 2018)

9. R.V. Kuzmenko, S.I. Moiseev, L.V. Stepanov, Proc. 2017 2nd Int. Ural Conf. on Measurements (UralCon) South Ural State University Chelyabinsk, 211 - 216 (2017)

10. T.A. Averina, S.A. Barkalov, S. I. Moiseev, Society, Integration, Education, Proc. of the Int. Scientific Conf. Rezekne Academy of Technologies VI, 42-52 (2018)

11. A.A. Maslak, G. Karabatsos, T.S. Anisimova, S.A. Osipov, Journal of Applied Measurement 6(4), 432 - 442 (2005) 